\title{
Particle Models of Discharge Plasmas in Molecular Gases
}

\author{
S. Longo, M. Capitelli, and P. Diomede \\ Dipartimento di Chimica dell'Universita' di Bari, Via Orabona 4, 70126 Bari, Italy \\ IMIP/CNR, Via Orabona 4, 70126 Bari, Italy \\ cscps117@area.ba.cnr.it
}

\begin{abstract}
We describe a mixed particle/continuum model for discharge plasmas in molecular gases developed by our group, which couples a particle description of the plasma phase with the diffusion/reaction kinetics of atoms and molecules in the gas phase. The model includes an improved treatment of ion kinetics, which fits some serious problems of multi time scale physical chemistry. The hydrogen plasma is considered as a test case. Results and computational costs are shortly discussed also in comparison with a different code.
\end{abstract}

\section{Introduction}

The modelling of the discharge plasma in molecular gases $[1,2]$ represents an important benchmark for the computational techniques of the plasma discharge community. Most of the models developed until now are 'fluid' models based on a macroscopic description of the charged particle kinetics in the discharge [3]. These models assume for the ionization coefficient, and in general for the rate coefficients of the electron/molecule reactions, values which are at most calculated by solving the so-called quasi-isotropic Boltzmann equation assuming a uniform plasma. These are later interpolated in terms of the electron energy and applied locally depending on the calculated value of the electron temperature. This approach cannot handle cases where the electrons have a Knudsen number higher than about 0.01 and behave like a rarefied gas. For a typical plasma dimension of $6 \mathrm{~cm}$, at room temperature, assuming an electron/neutral elastic cross section of $10^{-19} \mathrm{~m}^{2}$, the borderline pressure at which $\mathrm{Kn}=0.01$ is in the order of 0.5 torr. When the pressure is significantly lower than this, only methods based on numerical solutions of kinetic equations for the translational distribution function $f(\mathbf{r}, \mathbf{v}, t)$ of the charged particles, can be applied in order to get accurate values for the rate coefficients of electron/molecule reactions.

In general terms, the coupling of the neutral gas and plasma phase kinetics is always to be taken into account, especially for molecular gases: on one side in fact, the rate coefficients for electron/molecule reactions are functionals of the local electron energy distribution function; on the other side, the Boltzmann collision integral is also a function of the gas composition, including the vibrational excitation.

The necessity to take into account chemical kinetics as well as charged particle kinetics in the plasma phase at the same time creates an interesting problem of multiple time scales. In fact: 
1. the shortest electron particle kinetics time scale is the reverse of the electron plasma frequency, which in typical applicative cases is in the nanosecond range.

2. the time scale for charged particle relaxation to the steady state is the ambipolar diffusion time, which in typical applicative cases is of the order of 0.1-1 milliseconds

3. the time scale for the stabilization of a steady state chemical composition of the negative ions can reach (especially for electronegative plasmas) values of 0.01 seconds or higher.

So we have a time scale span of several orders of magnitude to be tackled by an appropriate technique, which must at the same time merge two different kinetic descriptions, one (for charged particles) based on transport kinetic equations, and the other (for neutral particles) based on chemical kinetics rate-diffusion equations.

Several particle models of these kind of plasmas already appeared in the literature [11], but as a rule they do not include the vibrational/chemical kinetics of gas phase molecules. A class of particle models including the kinetics of vibrationally excited molecules was considered in the past few years $[5,6]$. These models include a state-tostate chemical kinetics for molecules, this last term meaning that molecules in different vibrational levels are treated as separate species. Another important problem not yet addressed for this system is an accurate, kinetic-level description of the several kinds of ions existing in the discharge, which should take into account also the thermal distribution of neutral particles in the bulk region of the plasma, where it cannot be neglected because of the thermal energy of ions.

In this paper we present a fully self-consistent $1 \mathrm{D}$ model of the discharge plasma based on substantial improvements (to be described in details in the following) of previous models $[5,6]$.

The algorithm presented in this paper includes several techniques to treat the different time scales described in the introduction, in particular the self-consistent treatment of neutral chemistry and plasma kinetics and a special scaling for the negative ion time scale.

\section{Mathematical Statement of the Problem}

In reactive plasmas usually the relaxation times of the chemical kinetics are by far higher than the corresponding ones for the electron kinetics, therefore adiabatic elimination of the time dependence of the neutral component densities can be applied.

In the $1 \mathrm{D} \times 3 \mathrm{D}$ (meaning 1 space dimension and 3 velocity components) case, which can be reduced to $1 \mathrm{D} \times 2 \mathrm{D}$ unless transversal fields are applied, we can formalize our problem as follows:

$$
\left(\frac{\partial}{\partial t}+v_{x} \frac{\partial}{\partial x}-\frac{q_{s}}{m_{s}} \frac{\partial \varphi(x, t)}{\partial x} \frac{\partial}{\partial v_{x}}\right) f_{s}(x, \mathbf{v}, t)=C_{s}\left(\left\{F_{c}\right\}\right)
$$




$$
\begin{gathered}
\frac{\partial^{2} \varphi(x, t)}{\partial x^{2}}=-\frac{1}{\varepsilon_{0}} \sum_{s} q_{s} \int d^{3} v f_{s}(x, \mathbf{v}, t) \\
-D_{c} \frac{\partial^{2} n_{c}(x)}{\partial x^{2}}=\sum_{r}\left(v_{r c}^{\prime}-v_{r c}\right) k_{r}\left(\left\langle f_{e}\right\rangle_{t}\right) \prod_{c^{\prime}} n_{c^{\prime}}^{v_{r c}}
\end{gathered}
$$

where $f_{s}$ and $F_{c}$ are the kinetic distribution functions for the s-th charged species and the $\mathrm{c}$-th neutral species respectively, $\mathrm{f}_{\mathrm{e}}$ is the defined below (Eq.5), $\mathrm{q}_{\mathrm{s}}$ and $\mathrm{m}_{\mathrm{s}}$ are the $\mathrm{s}$-th species electric charge and mass, $\varphi$ is the electric potential, $\mathrm{n}_{\mathrm{c}}$ is the number density of the c-th neutral component, $\mathrm{D}_{\mathrm{c}}$ is its diffusion coefficient, $\mathrm{k}$ and $\mathrm{v}$ are, respectively, the rate coefficient and the molecularity of the c-th species in the r-th elementary process in a set of $\mathrm{N}_{\mathrm{r}}$

$$
\left\{\sum_{c=1}^{N_{c}} v_{r c} X_{c} \rightarrow \sum_{c=1}^{N_{c}} v_{r c}^{\prime} X_{c}\right\}_{1 \leq r \leq N_{r}}
$$

where $X_{c}$ is a generic neutral species out of a set of $N_{c}$.

We also include surface processes by an appropriate implementation of the boundary conditions [6].

In eq.(1a), $\mathrm{C}_{\mathrm{s}}$ is the Boltzmann collision integral for charged/neutral particle collisions:

$$
\begin{aligned}
& C_{s}\left(\left\{F_{c}\right\}\right)=-f_{s}(v) \int d^{3} v^{\prime} p_{v \rightarrow v^{\prime}}+\int d^{3} v^{\prime} p_{v^{\prime} \rightarrow v} f_{s}\left(v^{\prime}\right) \\
& p_{v^{\prime} \rightarrow v}=\int d^{3} w d^{3} w^{\prime}\left|v^{\prime}-w^{\prime}\right| \sum_{k} \sigma_{k}\left(v^{\prime}, w^{\prime}, v, w\right) F_{c(k)}\left(r, w^{\prime}\right)
\end{aligned}
$$

where $\mathrm{k}$ is an index addressing a specific collision process, $\sigma_{k}$ and $\mathrm{c}(\mathrm{k})$ are the differential cross section and the neutral collision partner of the $\mathrm{k}$-th process.

In the case of reactions including the electron as a reactant, the rate coefficient of the process must be written as (under the approximation of static neutral target):

$$
k(x)=\sqrt{2 / m_{e}} \int_{0}^{\infty} \varepsilon f_{e}(\varepsilon, x) \sigma(\varepsilon) d \varepsilon
$$

where $\sigma$ is the related total cross section and $\mathrm{f}_{\mathrm{e}}(\varepsilon, \mathrm{x})$ is the so-called electron energy distribution function (eedf) defined as

$$
\begin{aligned}
& f_{e}(\varepsilon, x)=\varepsilon^{-1 / 2}\left\langle f_{e}(x, \mathbf{v}, t) / n_{e}\right\rangle_{t, \Omega(\varepsilon)} \\
& \Omega(\varepsilon)=\left\{\mathbf{v} \in R^{3}, m_{e} v^{2}=2 \varepsilon\right\}
\end{aligned}
$$

this function is normalized to 1 for any position $\mathrm{x}$, and is usually measured in $\mathrm{eV}^{-3 / 2}$. The rate coefficient (eq.4) is a functional of the eedf and cannot be simply written as a 
function of the electron mean energy; this can only be done in the quasi-equilibrium case, where the eedf is maxwellian.

To fix ideas, in the following we will consider a code implementation for molecular hydrogen: we have selected this test case in view of the special status of hydrogen in physics and chemistry, and the several important applications of gas discharges in pure hydrogen and mixture of hydrogen with other gases. The code version for hydrogen includes several reactions and plasma phase processes. The species considered are five particle species in the plasma phase, i.e. electrons, $\mathrm{H}_{3}{ }^{+}$, $\mathrm{H}_{2}{ }^{+}, \mathrm{H}^{+}, \mathrm{H}^{-}$; and sixteen neutral components, i.e. $\mathrm{H}_{2}(\mathrm{v}=0$ to 14$)$ and hydrogen atoms.

A list of the collision and chemical processes is in ref.[6,(2001)]. The only changes are: the vibrational deactivation probability on the walls is here $\gamma_{\mathrm{V}}=0.1$, a mean between those used in the two refs.[6], the $\mathrm{H}^{-} / \mathrm{H}_{3}{ }^{+}$and $\mathrm{H}^{-} / \mathrm{H}$ reaction rates are after Gorse et al. [8], and the $\mathrm{H}_{3}{ }^{+} / \mathrm{H}_{2}$ cross section is from ref.[9].

\section{Numerical Method}

To solve the problem described in the previous sections we use a Particle in Cell/ Monte Carlo method (PIC/MC), which is a lagrangian/stochastic solution technique, for the transport equation and a grid-discretized relaxation technique for the reactiondiffusion part. A self-consistent approach involving joint solution of the two problems is necessary, for the reasons explained in the introduction. The procedure is repeated up to full relaxation.

In the PIC/MC approach the Newton equation for a large ensemble $\left(10^{4}-10^{5}\right.$ particles) of mathematical point particles (assumed to represent a statistically representative sample of electrons and positive ions) is solved taking into account the local electric field as it results from local interpolation within a cell of a mathematical mesh (from which follows the name 'Particle in Cell'). The electric charge is determined by sampling the particles on the mesh. The electric potential and field are determined by solving the Poisson equation on the same mesh (which in the 1D case is trivial [4]).

The PIC method delivers a solution of the Vlasov-Poisson plasma problem $(1 \mathrm{a}, \mathrm{b})$ in the following form:

$$
f_{S}(\mathbf{r}, \mathbf{v}, t)=\frac{W_{S}}{N_{S}} \sum_{p=1}^{N} S\left(\mathbf{r}-\mathbf{r}_{p}\right) \delta\left(\mathbf{v}-\mathbf{v}_{p}\right)
$$

$\mathrm{W}_{\mathrm{s}}$ is the ratio between real and simulated particles, and $\mathrm{S}(\mathrm{x})$ is the particle shape factor which describes the way particles are assigned to the mesh. A typical choice for $\mathrm{S}$ is the so-called 'Cloud-in-Cell' one, which in 1D is:

$$
S(x)=\left\{\begin{array}{c}
1-2 x / \Delta x, \quad 0 \leq x<\Delta x / 2 \\
1+2 x / \Delta x, \quad-\Delta x / 2 \leq x<0
\end{array}\right.
$$

The Newton equations in PIC are usually solved by using the Leapfrog method. 
We use a modified time step technique [4], where the particle dynamics evolves in time with an appropriate time step $\Delta \mathrm{t}$, while the time-to-next collision $\mathrm{t}_{\mathrm{c}}$ is an independent variable for any particle, which decreases during the free-flight. The exact time when $\mathrm{t}_{\mathrm{c}}(\mathrm{i})=0$ marks a new collision event.

As regards the inclusion of the collision term $\mathrm{C}_{\mathrm{s}}$, as it has been demonstrated [7], a stochastic calculation of the r.h.s. of eq.(3) in the von Neumann sense delivers directly and formally an improved version of the null-collision Monte Carlo method including the thermal distribution of neutrals.

In the plasma model the particle ensemble is represented by the set of numbers:

$$
\left\{\mathrm{i}, \mathrm{r}(\mathrm{i}), \mathrm{v}(\mathrm{i}), \mathrm{t}_{\mathrm{C}}(\mathrm{i}), \mathrm{s}(\mathrm{i})\right\}_{1 \leq i \leq N} \quad ; \quad N=\sum_{s} N_{s}
$$

Here $\mathrm{i}$ identifies the $\mathrm{i}$-th simulated particle, $\mathrm{r}(\mathrm{i})$ and $\mathrm{v}(\mathrm{i})$ are the position and velocity vectors assigned to the particle, $s(i)$ is the particle species and $t_{c}(i)$ is the time left to the next Monte Carlo collision event assigned only after a collision event by

$$
\begin{aligned}
& t_{c}(i)=-\frac{1}{\alpha_{s(i), \max }} \ln \eta \quad ; \quad 0<\eta \leq 1 ; f(\eta)=1 \\
& \alpha_{s, \text { max }}=\max _{\mathbf{r}, g<g_{\max }} g \sum_{k} n_{c(k)}(\mathbf{r}) \sigma_{k, t o t}(g)
\end{aligned}
$$

where $g$ is the relative speed, $g_{\max }$ is a physically grounded cutoff value for $\mathrm{g}$ and $\mathrm{s}$ is the particle species as above. Unphysical (null) events due to the fact that the collision frequency is $<\alpha_{s, \text { max }}$ are removed by the rejection method.

Disappearing particles are removed from the simulation by putting $s(i)=0$. The particle list is compressed at regular times by removing 'zero' particles.

If $\mathrm{N}$ exceeds $2 \mathrm{Nin}$, where Nin is its initial value, particles with odd $\mathrm{i}$ are removed and all $\mathrm{W}_{\mathrm{s}}$ are multiplied by 2 . If, instead, $\mathrm{N}<0.5 \mathrm{Nin}$, the particle ensemble is doubled and all $\mathrm{W}_{\mathrm{s}}$ are divided by 2 .

Recombination processes cannot fit the basic PIC/MC formalism since they involve two charged particles. We treat these processes as a combination of two first order ones, each including one of two particle species involved in the process, during a PIC time step $\Delta \mathrm{t}$. Charge neutrality is not enforced, but obtained on the average.

Two different values for the different $\mathrm{W}_{\mathrm{s}}$ are used by applying the technique introduced in ref. [6], in order to reduce the statistical error over macroscopic quantities calculated for minoritary ionic species, i.e. $\mathrm{H}_{2}^{+}, \mathrm{H}^{+}, \mathrm{H}^{-}$. In the calculation presented hereafter $W\left(H^{+}\right) / W(e)=0.1$ and the same for $\mathrm{H}_{2}^{+}$and $\mathrm{H}^{-}$.

Negative ions reach a steady state density arising from the balance of pseudo zero order production channel $\alpha$ (attachment) and pseudo first-order consumption channels $\beta n\left(\mathrm{H}^{-}\right)$(detachment, recombination), i.e. $\alpha=\beta \mathrm{n}\left(\mathrm{H}^{-}\right)$. The relaxation time $1 / \beta$ is often very long with respect to the other plasma time scales (about 0.01 seconds in the test case of the next section).

A special technique has been devised to speed up the convergence of the negative ion density: we scale both $\alpha$ and $\beta$ to $\mathrm{k} \alpha$ and $\mathrm{k} \beta$ respectively, with $\mathrm{k}>1$, while keeping $\mathrm{n}\left(\mathrm{H}^{-}\right)=\alpha / \beta$ constant. This implies scaling the cross sections for attachment 
and detachment, and the rate coefficients in the treatment of $\mathrm{H}^{-}$recombination (see above). The technique works since electrons and other ion densities are not affected significantly by attachment or $\mathrm{H}^{-}$detachment. This has been checked by direct comparison with code results with $\mathrm{k}=1$.

As regards the neutral kinetic equation (1c) we preliminarily linearize the loss term and rewrite the equation in the following form:

$$
\left(-D_{c} \frac{\partial^{2}}{\partial x^{2}}+\sum_{r} v_{r c} k_{r} \prod_{c^{\prime}} n_{c^{r^{\prime}}}^{v_{r c}-\delta_{c c^{c}}}\right) n_{c}(x)=\sum_{r} v_{r c}^{\prime} k_{r} \prod_{c^{\prime}}{ }_{c^{\prime}}^{v_{r c}}
$$

This procedure is convenient since in gas phase chemistry loss terms have mostly reaction order one in the lost species. Following space discretization, a Gauss-Seidel relaxation is applied to the resulting system of equations.

\section{Test Case: Radio Frequency Discharge in Hydrogen}

As a test case, we consider a pure hydrogen RF discharge plasma produced by the parallel-plate configuration, i.e. one plane electrode surface $(x=0)$ is kept at electric potential $V=0$ (grounded), while the opposite one $(x=d)$ is assumed to be driven by an external generator to an oscillating potential.

The PIC mesh has 400 cells, while the neutral reaction/diffusion equation mesh has 50 cells. The PIC time step is $0.05 \mathrm{~ns}$, fitting the Courant condition.

The code was run for $10^{6} \mathrm{PIC}$ time steps with $5 \mathrm{PIC} /$ kinetics iterations in order to reach a steady state solution. The main bottleneck is the (albeit scaled) negative ion density relaxation.

The values for physical quantities are: gas temperature $\mathrm{T}_{\mathrm{g}}=300 \mathrm{~K}$, voltage amplitude $200 \mathrm{~V}$, gas pressure $p=0.1$ torr, discharge frequency $=13.56 \mathrm{MHz}$, discharge gap $d=0.06 \mathrm{~m}$, DC voltage (bias) $=0 \mathrm{~V}$.

Figs.1-4 show a snapshot of the results. In particular:
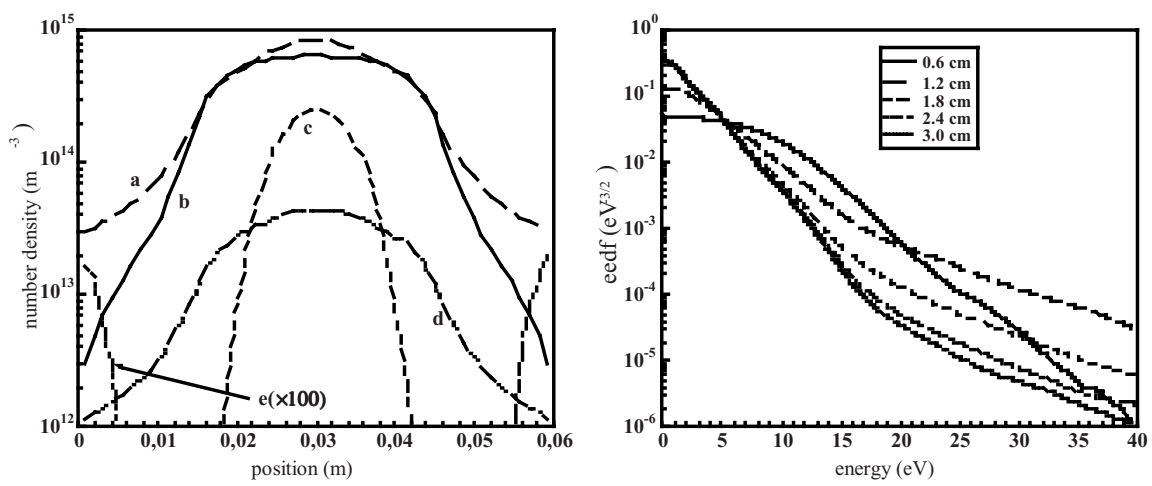

Fig. 1. Left: Number density of charged particles at the steady state for the test case: (a) $\mathrm{H}_{3}{ }^{+}$ ions, (b) electrons, (c) $\mathrm{H}^{-}$ions, (d) $\mathrm{H}^{+}$ions, (e) $\mathrm{H}_{2}{ }^{+}$ion; right: the eedf at different positions in the discharge. 
Fig. 1 shows the results obtained for the number densities of charged particles. It can be seen that the role of negative ions in the central region of the plasma is not negligible, contrary to the implicit assumption of most of the literature in the field. A large difference is observed for what regards the negative ion density in Fig.1 with respect to ref.[6] due to the different value of $\gamma_{\mathrm{v}}$.
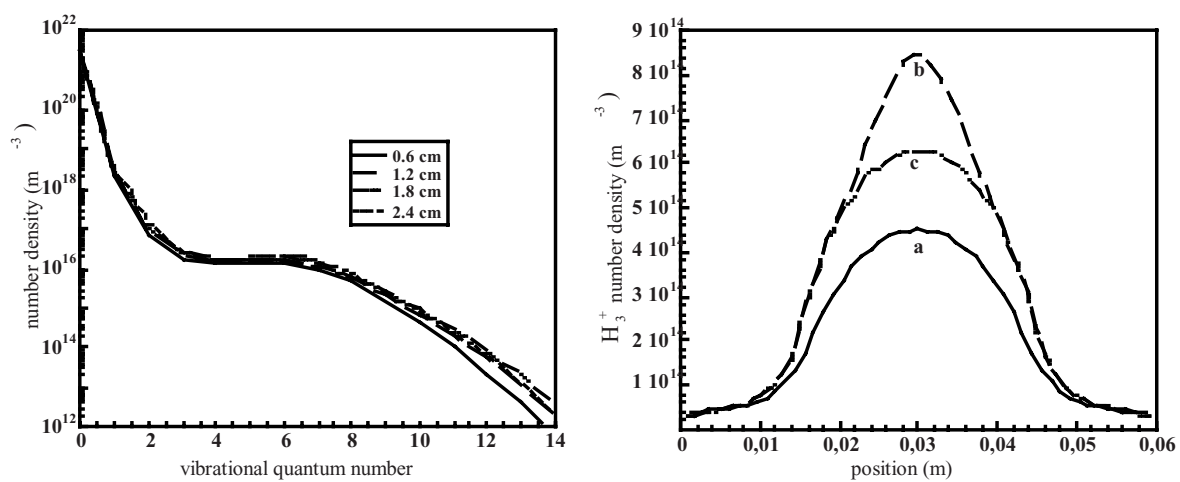

Fig. 2. Left: Plot of the vdf at different positions in the discharge; right: Comparison between $\mathrm{H}_{3}{ }^{+}$number density, as a function of position, calculated by SIGLO-RF fluid model code (a) and our PIC/MC model taking into account (b) and neglecting (c) $\mathrm{H}^{-}$ion production

Figs. 1-right/2-left show the eedf and the vibrational distribution function (vdf) in different positions in the discharge. For both it is confirmed a non equilibrium character. As already well known the vdf is characterised by a plateau due to EV excitation processes (i.e. high threshold processes with a singlet intermediate [1]) occurring in the sheath/bulk boundary region where the electron energy deposition is larger.

Fig.2-right shows a comparison between our PIC/MC code results and those obtained with the fluid modelling based SIGLO-RF [10] code in the same physical conditions and using mostly the same input data. This code, however, neglects the negative ion and the vibrational kinetics.

It can be noticed a good agreement for the density of the majority positive ion.

The comparison is better in the case of the code run which neglects negative ions, as expected.

These results could be obtained at the cost of 10.5 hours on a Pentium $4,1.7 \mathrm{GHz}$ PC. The most remarkable point on this respect is the success of the negative ion time scale technique, which allowed to reduce the CPU time by a factor of ten without affecting the overall results in any sensible way.

Since there is no need for a particle/mesh list, the approach is not only highly suitable for parallelization but possibly, with some modifications, for a GRID network implementation.

Of course the computational cost of a fluid model run is by far lower, but this last is less informative as regards the electron energy distribution, since it considers local field conditions based on the calculated local electron energy, and therefore cannot 
reproduce kinetic tails, like in Fig.2, which are due to fast electron diffusion. Besides, for the same reason, the fluid approach is not applicable to more rarefied cases, which are easily accessed by particle models like the one presented here.

\section{Conclusions}

A one dimensional particle model for non equilibrium plasma developed in the past has been applied to hydrogen and improved as regards the treatment of ion kinetics.

In particular we have added a kinetic description of the interaction of charged particles with the thermal distribution of gas particles, a weighting method which enhances the statistics of minoritary ionic species and a scaling technique to speed up the negative ion stabilization. The new version of the method can deliver a very detailed view of the plasma kinetics at a reasonable computational cost.

Acknowledgments. This work has been partially supported by ASI (contr. I/R/055/02) and FISR "Modellistica Molecolare di Sistemi di complessita' Crescente"

\section{References}

1. M.Capitelli, C.M.Ferreira, F.Gordiets, A.I.Osipov, 'Plasma Kinetics in Atmospheric Gases', Springer Verlag 2000; M. Capitelli (Ed.), Non Equilibrium Vibrational Kinetics, Springer, New York, 1985

2. M.A.Liebermann and A.J.Lichtemberg, 'Principles of Plasma Discharges and Materials Processing', Wiley N.Y. 1994

3. A.Salabas, G.Gousset and L.L.Alves, Plasma Sources Sci.Techn. 11448 (2002); J.P.Boeuf and Ph. Belenguer, J.Appl.Phys. 714751 (1992)

4. C. K. Birdsall, IEEE Trans. Plasma. Sci. 19 68-85 (1991); R. W. Hockney and J. W. Eastwood, 'Computer Simulation Using Particles', Adam Hilger 1991

5. S. Longo, K. Hassouni, D. Iasillo, and M. Capitelli, J. Phys. III 7707 (1997); S. Longo, M. Capitelli and K. Hassouni, J. Phys. IV C4 271 (1997); S. Longo, M. Capitelli, K. Hassouni, Journal of Thermophysics and Heat Transfer 12473 (1998)

6. S. Longo, I. D. Boyd, Chem. Phys. 238445 (1998), S. Longo, A. Milella, Chem. Phys. 274219 (2001).

7. S. Longo, Physica A 313389 (2002); S. Longo, P. Diomede, Eur.Phys.J AP, in press

8. C. Gorse, R. Celiberto, M. Cacciatore, A. Laganà, M. Capitelli, Chem. Phys. 161 (1992) 211

9. T. Simko, V. Martisovits, J. Bretagne, G. Gousset, Phys. Rev. E 565908 (1997)

10. J.P. Boeuf, L.C. Pitchford, 1995, SIGLO-RF, PC version 1.0, a 1D User-Friendly Model for RF Discharges Simulation (Kinema Software, siglo@kinema.com, http://www.siglokinema.com/siglo-rf.htm, Monument, CO)

11. M. Yan, A. Bogaerts, W. J. Goedheer and R. Gijbels, Plasma Sources Sci. Techn. 9583 (2000), K.Radouane et al., J.Appl.Phys. 904346 (2001). 